\title{
Variational principles are a powerful tool also for formulating field theories
}

\author{
Francesco dell'Isola ${ }^{* \ddagger}$ and Luca Placidi ${ }^{\dagger \ddagger}$ \\ * University of Roma "La Sapienza" \\ † International Telematic University "Uninettuno" \\ ¥ International Research Centre on "Mathematics \& Mechanics of Complex \\ Systems" M\& MOCS
}

\begin{abstract}
Variational principles and calculus of variations have always been an important tool for formulating mathematical models for physical phenomena. Variational methods give an efficient and elegant way to formulate and solve mathematical problems that are of interest for scientists and engineers and are the main tool for the axiomatization of physical theories.
\end{abstract}

\section{Introduction and historical background}

\subsection{Metrodoron and his followers}

The ideas we want to evocate in this lecture are very old and were put forward already in the hellenistic period: for a detailed discussion about this point the reader is referred to the beautiful book by Lucio Russo (2003). In that book it is established that "modern" science actually was born in the hellenistic era, when Metrodoron lived. Metrodoron was a pupil of a famous greek philosopher, Epicurus, and, in our opinion, the following Metrodoron's sentence is a statement (the first?) belonging to the modern philosophy of science:

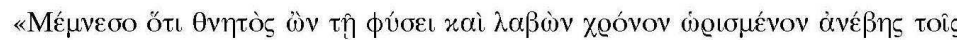

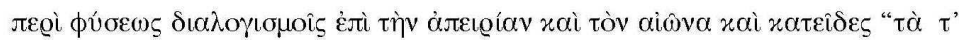

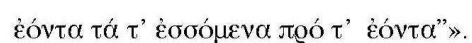

\section{Metrodoron,}

"Always remember that you were born mortal and such is your nature and you were given a limited time: but by means of your reasonings about Nature you could rise to infinity and to eternity 
and you indeed contemplate "the things that were, and that were to be, and that had been before"". Metrodoron

Gnomologium Epicureum Vaticanum X (fr.37 Alfred Körte, Metrodori Epicurei Fragmenta, " Jahrbücher für classiche Philologie", Suppl. XVII, 1890, p. 557).

This dictum, following Körte, comes from a lost letter or book by Metrodoron (the Epicurean philosopher) addressed to Menestratos who was presumably one of his pupils. The words quoted in bold are a citation from Iliad, I 70 (the translation into English of the sentence in boldface is ours; except for this citation the translation has been taken from Homer by Murray (1924), see the ref. (14)).

In different words, Metrodoron states that by using (the right!) equations you can forecast future behavior of physical systems.

\subsection{Why Variational Principles and Calculus of Variation?}

In recent time, a lost Archimedes' book (19) has been rediscovered. Some authors claim that Archimedes seems to have solved, in this book and using a variational principle, the technological problem of finding the optimal shape of a boat. Archimedes seems to have chosen, as optimality criterion, that the vertical position must be a "very" stable configuration (see Rorres (2004)). In the book of Russo (21) it is demonstrated in even a more convincing way that many optimization techniques were well-known in hellenistic science. In particular Russo proves that the problem of the determination of the regular polygon having maximal area has been solved in that period. Thus, the use of a variational principle and optimization methods to solve technological problems is less recent than it is usually believed. In general, variational formulation of the governing equations of solid and fluid mechanics is a classical but very challenging topic. This kind of formulation allows for an easier proof of the well-posedness of mathematical problems, for an easier investigation of the study of stability of particular solutions, and for a simpler implementation of numerical methods. Often (but one who believes in Russo's vision about the birth of science could say instead "always"), mechanical problems are more naturally posed by means of variational methods. Hamilton's principle of stationary (or least) Action is the conceptual basis of practically all models in physics. The variational formulation is also useful for obtaining simpler approximate asymptotical models as it is done in the theory of homogenization.

We want simply to state here that the Principle of Virtual Works and the Principle of Least Action have roots much deeper than many scientists believe (see Vailati, 1897). Although many histories of science claim dif- 
ferently, most likely the majority of physical theories were first formulated in terms of these Principles, and only subsequently they were reconsidered from other points of view. In our opinion the Principle of Least Action, which supplies a "geometric" version of mechanics was indeed the tool used by the true founders of mechanics (i.e. the scientists of the hellenistic period) to establish it. As argued also by Colonnetti (5) and Netz and Noel (19)) surely also Archimedes and ancient greek scientists were accepting such a point of view.

The epigones of the hellenistic science, who were not able to understand the delicate mathematical arguments used by the first scientists, however could understand the minimality conditions obtained by their "maitres" (i.e. the conditions corresponding to those which we call now Euler-Lagrange equations and boundary conditions) and could grasp the "physical" arguments used to interpret them. This phenomenon is perfectly clear to everyone who is ready to consider carefully -for instance- the evolution of the theory of Euler-Bernoulli Beam (a useful reference about this point is the book of Benvenuto (1981)). Euler postulated a Principle of Least Action for the Elastica, and gets the celebrated equilibrium differential equation and boundary conditions for the equilibrium of beams by using the calculation procedure due to Lagrange (which is the departing idea of Calculus of Variations). Then Navier prepared his lectures for the Ecole Polytechnique and resumed the results obtained by Euler deciding to "spare" to the (engineering) students the difficulties of the calculus of variations. He started directly from the equilibrium equation, obtained by means of an "ad hoc" principle of balance of force and couple, and imposed boundary conditions based on "physical" assumptions. As a consequence, for a long while, generations of engineers believed that the beam equations were to be obtained in this way. Only when numerical simulations became popular, then they (actually, some of them) became aware of variational "principles". However these principle were proven as theorems starting from "balance postulates" and were considered simply as a mathematical (rather abstruse) tool and not as a fundamental heuristic concept. And this attitude is not changed even when it became clear that every serious advancement of mechanical science has been obtained using variational principles. Indeed the so called "physical sense" (a gift that many claim to posses but which nobody can claim to be able to master or to teach) is not very useful to postulate the right "balance principles" when one is in "terra incognita". For instance, when Lagrange and Sophie Germain wanted to find the plate equations they needed to employ a variational principle (and they could find the (right!) natural boundary conditions). Again when Cosserat brothers wanted to improve Cauchy Continuum Mechanics they "rediscovered" the right tech- 
nique: i.e. the Principle of Least Action. Also Quantum Mechanics has been developed starting from a Variational Principle (see e.g. the references of Feynman (11), Lagrange (15) and Lanczos (16)).

Therefore an important warning is due to young researcher: refrain from trying to extend available models by means of "ad hoc" adaptations of available theories: always look for the right Action functional to be minimized!

\subsection{The problem of including dissipation}

One useful tool for handling complicated situations is used in Continuum Mechanics by Paul Germain when formulating second gradient theories: the Principle of Virtual Powers. Again, as remarked always in the history of the development of ideas, when this history can be reconstructed, the effective way to be used to proceed is that which starts from a Principle of Least Action, eventually generalized into a Principle of Virtual Powers. For a long time the opponents to Second Gradient Theories argued about its lack of consistency, due to the difficulties they claim to find in "getting" boundary conditions. This is a really odd statement. Indeed variational principles easily produce mathematically correct boundary conditions. So maybe what those opponents want to say is that as they are not so clever as Navier, they do not manage to interpret physically the boundary conditions found via a (correct and meaningful) variational principle. Of course if one refuses to use the Principle of Least Action he can find very difficult the job of determining some set of boundary conditions which are compatible with the (independently postulated!) bulk evolution equations. If instead one accepts the Archimedean (the reader will allow us to dream, without definitive evidence that such was the point of view of Archimedes) approach to mechanics then all these problems of well-posedness of mathematical models completely disappear.

Variational Principles always produce intrinsically well-posed mathematical problems, if the Action functional is well behaving. Of course passing from Lagragian systems (the evolution of which are governed by a Least Action functional) to non-Lagragian systems (for which such a functional may not exist) maybe difficult. This problem is related (but is not completely equivalent) to the problem of modelling dissipative phenomena. It is often stated that dissipation cannot be described by means of a Least Action Principle. This is not exactly true, as it is possible to find some Action functionals for a large class of dissipative systems. However it is true that not every conceived system can be regarded as a Lagragian one. This point is delicate and will be only evocated here. In general a non-Lagragian system can be regarded as Lagragian in two different ways: i) because it 
is an "approximation" of a Lagrangian system (see the case of Cattaneo equation for heat propagation), and this approximation leads to "cancel" the lacking part of the "true" Action Functional ii) because the considered system is simply a subsystem of a larger one which is truly Lagrangian. The assumption that variational principle can be used only for non-dissipative systems is contradicted by, e.g., the work presented in this book by Prof. Frankfort (12), where you find variational principles modelling dissipative systems. Indeed it is often stated that a limit of the modelling procedure based on variational principles consists in their impossibility of encompassing "nonconservative" phenomena. We do not believe that this is the case: however in order to avoid to be involved in a problem which is very difficult to treat, when dealing with dissipative systems, we will assume a slightly different point of view, usually attributed to Hamilton and Rayleigh.

\section{Finding a mathematical model for natural phenomena}

\subsection{Principle of Least Action}

We want to discuss here about the problem of finding a mathematical model for natural phenomena. We start with an epistemological Principle:

"The Principle of Least Action tells us how to construct a mathematical model to be used for describing the world and for predicting the evolution of the phenomena occurring in it".

In the following modeling scheme, we give the right heuristic strategy to be used for finding an effective model using the Principle of Least Action. The recipe includes the following ingredients:

1. Establish the right kinematics needed to describe the physical phenomena of interest, i.e. the kinematical descriptors modeling the state of considered physical systems.

2. Establish the set of admissible motions for the system under description, i.e. establish the correct model for the admissible evolution of the system.

3. Employ the "physical intuition" to find the right Action functional to be minimized, i.e. modeling what Nature wants to minimize.

We start by finding the kinematical descriptors, because of the need of modeling the states of the considered system. Then we introduce motion, in such a way we model the evolution of the system to be described. Finally we ask Nature what is the quantity to minimize. Keeping this quantity in mind, we introduce the Action functional. To start with, it is necessary 
to focus the attention on a specific class of systems and on phenomena occurring to them. A configuration is the mathematical object used to model the state of considered systems: the set of possible configurations will be denoted by $C$. The motion is the mathematical model describing the evolution of considered systems: it is a $C$-valued function defined on time interval $\left(t_{0}, t_{f}\right)$; the set of all admissible motions will be denoted by $M$. The Action is a real-valued function, defined on $M$, which models the "preferences" of nature.

Finally, to use the Principle of Least Action one needs three steps further,

4. Find the Euler-Lagrange conditions which are consequence of the postulated Least Action Principle

5. Interpret these condition on a physical ground

6. Determine, in terms of the postulated Action functional, the numerical integration scheme to be used to get the previsions needed to drive, by means of our theory, our experimental, technological or engineering activity.

\subsection{The Rayleigh-Hamilton principle}

When postulating an extended Rayleigh-Hamilton principle, the point 4 of the previously presented heuristic strategy will be further divided into two steps as follows:

4a. Once the quantities which expend power on the considered velocity fields are known in terms of postulated Action, introduce a suitable definite positive Rayleigh dissipation functional

4b. Equate the first variation of Action functional to the Rayleigh dissipation functional and get the evolution equations (including boundary conditions) which govern the motion of the system

Although in the literature the choice of including a Rayleigh-Hamilton principle in the class of variational principles is sometimes considered inappropriate, we will follow what seems to us the preference of the majority of the authors: therefore we do call "variational" also the strategy which we just described, not limiting the use of this adjective to the models using exclusively the Least Action Principle.

\subsection{La Cinèmatique d'Abord !}

According to Metrodoron, mathematical and physical objects are two different concepts. Indeed, equations are necessary for modeling physical systems but they refer to mathematical objects. When one solves the equations formulated in the framework of his model then he has to transform the 
obtained equations into previsions valid for the physical system he is studying. A good modeling procedure uses mathematics for finding the motion which minimizes Action. If this mathematics gives a reasonable forecasting of the observed evolution, then the model is valid. However, not everything is described by a given model. A model is always focused on a set of phenomena.

The set of phenomena that are focused by a model is established by the kinematics:

\section{La Cinèmatique d'Abord !}

In the previous scheme it is clear that the most "fundamental" step concerns the choice of the set of configurations used for characterizing the "accessible" states of the system. When constructing a mathematical model using the discussed epistemological principle, one must start with a precise and clear determination of the set $C$. The second step concerns the determination of admissible motions which clearly depend on the evolutionary phenomena one wants to model. A correct modeling process always starts specifying "admissible" kinematics.

\subsection{La Nature agit toujours par les voies les plus simples}

After having specified the admissible kinematics, one can wonder about the desire of Nature. The utility of Nature is a real-valued function defined on $M$. Following Maupertuis, we will call Action this "utility". Also Nature must consider which is the contingent situation: not all admissible motions are accessible by a physical system under given specific conditions. Therefore we must specify a subset $M_{A}$ of the set $M$ : the set of accessible motions. The real motion will be chosen by the system minimizing the Action in the subset $M_{A}$. Indeed:

La Nature agit toujours par les voies les plus simples.

\subsection{Two possible choices for the set of admissible and accessible motions}

In the famous textbook of Arnold (1) the author, following the tradition, does not "try" to explain Maupertuis' Principle of Least Action. We instead dare to try to deal with this. In the process of minimization of the Action, we need to specify the set of motions among which we look for minima. The choice of Lagrange is that of isochronous motions. Two motions are isochronous when they both start, at the given instant $t_{0}$, from a given configuration $C_{0}$ and arrive, at the same instant $t_{f}$, at the same final configuration $C_{f}$. On the other hand, the choice of Maupertuis is to focus 
on the set of motions with a "fixed energy content" and which are starting from the same configuration $C_{0}$ and ending (the instants of start and stop are not specified!) at the configuration $C_{f}$. In the set of admissible motions an "energy" functional must then be introduced: i.e. a functional which associates an energy content to any motion and any time instant $t$. The set of accessible motions is constituted by all motions from $C_{0}$ to $C_{f}$ which have a constant energy content. The choice of Maupertuis, if not suitably modified, seems to limit the range of applicability of variational principles to non dissipative phenomena.

\subsection{Further famous quotes}

Many books in Calculus of Variations and/or Variational Principles, see e.g. that of Lanczos (1970), start with a preface, introduction or introductory chapter dealing with historical prolegomena and sometimes end with a philosophical chapter. In presenting this lecture notes, we did not dare to break with tradition.

"For this would be agreed by all: that Nature does nothing in vain nor labours in vain". Olympiodorus, Commentary on Aristotle's ${ }^{\mathrm{TM}}$ Meteora translated by Ivor Thomas in the Greek Mathematical Works Loeb Classical Library

"La nature, dans la production de ses effets, agit toujours par les voies les plus simples". Pierre de Fermat.

Now, the problem is:

What is utility?

\section{In other words: How to find "Real Motions"?}

Up to now no mathematical structure has been assumed for $M_{A}$. Indeed, Action functional is simply a real-valued map defined on $M_{A}$. "Practical" problems require the calculation of real motions by means of introduced model. Following Lagrange (15), we introduce a particular class of Action functionals in terms of a Lagrangian Action density function: so constructing in a particular way Action functional to obtain so called "Lagrangian functionals".

We need to introduce a topological structure in $M_{A}$, i.e. we need to clearly define what we mean when we say that "two motions are close". If we want to find minima of a real-valued function, then we need to estimate derivatives and equate these derivatives to zero. Action is a function defined in the set of motions (not real numbers!). Thus, we need 
- to understand what is an infinitesimal variation of motion,

- to find a differential of a functional and

- to estimate the order of infinitesimal of its remainder.

In other words, we need to learn how to find a first order Taylor expansion for a Lagrangian functional by establishing the meaning of the expressions :

- Infinitesimal variation of motion.

- Differential of a functional.

- Order of infinitesimals for remainders.

This implies the need of Frechèt and Gateaux derivatives in manifolds with charts in Banach spaces. This is the right mathematical frame for studying this subject. However, Lagrange did not know that he was using such a mathematical frame and did not know anything about Frechèt and Gateaux derivatives. Thus, in this notes we try to go around the related mathematical difficulties and follow the original approach of Lagrange.

The motion minimizing Action will be searched among the motions for which the first variation of Action vanishes.

For Lagrangian functionals this condition is equivalent to a partial differential equation which is called Euler-Lagrange condition relative to the given Action functional. This procedure generalizes the corresponding one used for real-valued functions of several real variables. One serious problem with papers that start from balance equations and "play" with forces is that they do not "find" boundary conditions. In these references $((7 ; 8 ; 9))$ one can find examples of modelling procedures in which one finds simultaneously bulk and boundary conditions.

From an historical point of view, in the theory of beam we deal with contact actions (normal and shear forces and momenta) because Navier has written lecture notes for l'Ecole Polytechique, trying to produce a text for students that was as simple as possible. He wrote final equations and explain not only bulk but also boundary conditions with the aid of "physical sense". However, it is very difficult in general to find evolution equations and boundary conditions with physical sense. On the other hand, variational principles give boundary conditions automatically and without the help of any physical sense.

Thus, Variational Principles allow Science to unveil Nature and for unveiling Nature you need a Lagrangian functional.

\section{Lagrangian Action Functionals: technical details}

We follow Landau and Lifshitz (1977) and Moiseiwitsch (1966). 
Let $\Psi_{\sigma}\left(x_{\mu}\right)$ be any set of $n$ tensor fields defined on $\mathbb{R}^{m}$, ( $\sigma$ being a multi-index and $\mu=1,2, \ldots, m)$. We define the Lagrangian density as:

$$
\mathfrak{L}\left(x_{\mu}, \Psi_{\sigma}, \frac{\partial \Psi_{\sigma}}{\partial x_{\mu}}\right) .
$$

We can then introduce the Action functional as

$$
\mathfrak{A}=\int_{T} \mathfrak{L}\left(x_{\mu}, \Psi_{\sigma}, \frac{\partial \Psi_{\sigma}}{\partial x_{\mu}}\right)
$$

Where $T$ is a hyper-volume in the $m$-th dimensional space determined by the coordinates $x_{\mu}$. When we will want to derive the theory of second gradient materials, this approach will not be appropriate, because we would need to add the dependence on the second gradient of $\Psi_{\sigma}$ in (1).

\subsection{Variation of the Action Functional}

We now consider small variations $\varepsilon \eta_{\sigma}\left(x_{\mu}\right)$ of the considered fields $\Psi_{\sigma}\left(x_{\mu}\right)$ :

$$
\tilde{\Psi}_{\sigma}\left(x_{\mu}\right)=\Psi_{\sigma}\left(x_{\mu}\right)+\varepsilon \eta_{\sigma}\left(x_{\mu}\right),
$$

where the $\eta_{\sigma}\left(x_{\mu}\right)$ are any set of linearly independent functions of the $x_{\mu}$ which vanish on the part $\partial_{d} T\left(\partial_{d} T \subseteq \partial T\right)$ of the boundary $\partial T$ of the hypervolume $T$, on which the kinematical condition are prescribed. The variation of the Action functional can then be computed as:

$$
\Delta \mathfrak{A}=\int_{T} \mathfrak{L}\left(x_{\mu}, \tilde{\Psi}_{\sigma}, \frac{\partial \tilde{\Psi}_{\sigma}}{\partial x_{\mu}}\right)-\int_{T} \mathfrak{L}\left(x_{\mu}, \Psi_{\sigma}, \frac{\partial \Psi_{\sigma}}{\partial x_{\mu}}\right)
$$

where $T$ is a hyper-volume in the $m$-th dimensional space determined by the $x_{\mu}$. The computation of the variation of the Action functional now proceeds as follows:

$\Delta \mathfrak{A}=\int_{T} \mathfrak{L}\left(x_{\mu}, \Psi_{\sigma}+\varepsilon \eta_{\sigma}, \frac{\partial \Psi_{\sigma}}{\partial x_{\mu}}+\varepsilon \frac{\partial \eta_{\sigma}}{\partial x_{\mu}}\right)-\int_{T} \mathfrak{L}\left(x_{\mu}, \Psi_{\sigma}, \frac{\partial \Psi_{\sigma}}{\partial x_{\mu}}\right)+O\left(\varepsilon^{2}\right)$

which, with a slight abuse of notations, can be written at the first order in $\varepsilon$ as:

$$
\delta \mathfrak{A}=\varepsilon \int_{T} \sum_{\sigma}\left(\frac{\partial \mathfrak{L}}{\partial \Psi_{\sigma}} \eta_{\sigma}+\sum_{\mu=1}^{m} \frac{\partial \mathfrak{L}}{\partial\left(\partial \Psi_{\sigma} / \partial x_{\mu}\right)} \frac{\partial \eta_{\sigma}}{\partial x_{\mu}}\right)
$$


Integrating by parts and recalling that $\eta_{\sigma}$ vanish on $\partial_{d} T$ it is easy to get:

$$
\begin{array}{r}
\delta \mathfrak{A}=\varepsilon \int_{T} \sum_{\sigma} \eta_{\sigma}\left(\frac{\partial \mathfrak{L}}{\partial \Psi_{\sigma}}-\sum_{\mu=1}^{m} \frac{\partial}{\partial x_{\mu}}\left(\frac{\partial \mathfrak{L}}{\partial\left(\partial \Psi_{\sigma} / \partial x_{\mu}\right)}\right)\right) \\
+\varepsilon \int_{\partial T / \partial_{d} T} \sum_{\sigma} \eta_{\sigma} \sum_{\mu=1}^{m} \frac{\partial \mathfrak{L}}{\partial\left(\partial \Psi_{\sigma} / \partial x_{\mu}\right)} N_{\mu},
\end{array}
$$

where $\partial T / \partial_{d} T$ is the difference between $\partial T$ and $\partial_{d} T$ and $N_{\mu}$ is the external unit normal of $\partial T / \partial_{d} T$. Imposing $\delta \mathfrak{A}=0$, the arbitrariness of $\eta_{\sigma}$ gives, for any $\sigma$ :

$$
\begin{array}{r}
\frac{\partial \mathfrak{L}}{\partial \Psi_{\sigma}}-\sum_{\mu=1}^{m} \frac{\partial}{\partial x_{\mu}}\left(\frac{\partial \mathfrak{L}}{\partial\left(\partial \Psi_{\sigma} / \partial x_{\mu}\right)}\right)=0, \quad \forall x_{\mu} \in T, \\
\sum_{\mu=1}^{m} \frac{\partial \mathfrak{L}}{\partial\left(\partial \Psi_{\sigma} / \partial x_{\mu}\right)} N_{\mu}=0, \quad \forall x_{\mu} \in \partial T / \partial_{d} T .
\end{array}
$$

In the case of a discontinuity material surface $\Sigma$ (with unit normal $N_{\mu}$ ) the (9) have to be completed by

$$
\sum_{\mu=1}^{m}\left[\left|\frac{\partial \mathfrak{L}}{\partial\left(\partial \Psi_{\sigma} / \partial x_{\mu}\right)}\right|\right] N_{\mu}=0, \quad \forall x_{\mu} \in \Sigma,
$$

where $[|(\cdot)|]$ is the jump of $(\cdot)$ across the surface $\Sigma$. These equations are known as the Euler-Lagrange equations corresponding to the considered Lagrangian density.

\subsection{The Space-Time Case $(m=4)$}

Let us now consider the particular case in which $m=4$. This case corresponds, for instance, to the case $x_{\mu}=\left(x_{1}, x_{2}, x_{3}, t\right)$. We have that $\eta_{\sigma}\left(x_{\mu}\right)$ are any set of linearly independent functions of the $x_{\mu}$ which vanish on the boundary of time type domain,

$$
\eta_{\sigma}\left(x_{1}, x_{2}, x_{3}, t_{0}\right)=\eta_{\sigma}\left(x_{1}, x_{2}, x_{3}, t_{1}\right)=0
$$

and on the part $\partial_{d} V$ of the boundary $\partial V$ of the volume $V$, on which the kinematical conditions are prescribed,

$$
\eta_{\sigma}\left(x_{1}, x_{2}, x_{3}, t\right)=0, \quad \forall\left(x_{1}, x_{2}, x_{3}\right) \in \partial_{d} V, \quad \forall t \in\left[t_{0}, t_{1}\right] .
$$


It is easy to show that in this particular case eq. (7) yields

$$
\begin{aligned}
\delta \mathfrak{A}=\varepsilon \int_{t_{0}}^{t_{1}} d t \int_{V} \sum_{\sigma} \eta_{\sigma}\left[\frac{\partial \mathfrak{L}}{\partial \Psi_{\sigma}}-\sum_{k=1}^{3} \frac{\partial}{\partial x_{k}}\left(\frac{\partial \mathfrak{L}}{\partial\left(\partial \Psi_{\sigma} / \partial x_{k}\right)}\right)\right. \\
\left.-\frac{\partial}{\partial t}\left(\frac{\partial \mathfrak{L}}{\partial\left(\partial \Psi_{\sigma} / \partial t\right)}\right)\right] \\
+\varepsilon \int_{\partial V / \partial_{d} V} \sum_{\sigma} \eta_{\sigma} \sum_{k=1}^{3} \frac{\partial \mathfrak{L}}{\partial\left(\partial \Psi_{\sigma} / \partial x_{k}\right)} N_{k} \\
+\int_{\Sigma} \sum_{\sigma}\left[\left|\eta_{\sigma} \sum_{k=1}^{3} \frac{\partial \mathfrak{L}}{\partial\left(\partial \Psi_{\sigma} / \partial x_{k}\right)}\right|\right] N_{k}
\end{aligned}
$$

The stationarity $\delta \mathfrak{A}=0$ of the Action implies, for any $\sigma=1,2, \ldots, n$,

$$
\begin{array}{r}
\frac{\partial \mathfrak{L}}{\partial \Psi_{\sigma}}-\sum_{k=1}^{3} \frac{\partial}{\partial x_{k}}\left(\frac{\partial \mathfrak{L}}{\partial\left(\partial \Psi_{\sigma} / \partial x_{k}\right)}\right)-\frac{\partial}{\partial t}\left(\frac{\partial \mathfrak{L}}{\partial\left(\partial \Psi_{\sigma} / \partial t\right)}\right)=0, \quad \forall x_{k} \in V, \\
\sum_{k=1}^{3} \frac{\partial \mathfrak{L}}{\partial\left(\partial \Psi_{\sigma} / \partial x_{k}\right)} N_{k}=0, \quad \forall x_{\mu} \in \partial V / \partial_{d} V, \\
\sum_{k=1}^{3}\left[\left|\frac{\partial \mathfrak{L}}{\partial\left(\partial \Psi_{\sigma} / \partial x_{k}\right)}\right|\right] N_{k}=0, \quad \forall x_{\mu} \in \Sigma .
\end{array}
$$

Which are the standard Euler-Lagrange equations. We will see in the next chapters of this book how to generalize (14) when $\Sigma$ can move freely.

\section{Principle of Virtual Power and Principle of Least \\ Action}

The principle of least Action, when formulated for Action functionals admitting first differentials, can be regarded as a particular form of the principle of virtual powers. Indeed, if

$$
\mathfrak{A}=\mathfrak{A}^{\text {int }}+\mathfrak{A}^{e x t}+\mathfrak{A}^{\text {ine }}
$$

then

$$
\delta \mathfrak{A}=0 \quad \Longleftrightarrow \quad \delta \mathfrak{A}^{i n t}+\delta \mathfrak{A}^{e x t}+\delta \mathfrak{A}^{i n e}=0
$$

Identifying

$$
\delta \mathfrak{A}^{\text {int }}=\mathfrak{P}^{\text {int }} \quad \delta \mathfrak{A}^{\text {ext }}=\mathfrak{P}^{e x t}, \quad \delta \mathfrak{A}^{\text {ine }}=\mathfrak{P}^{\text {ine }},
$$


we get

$$
\mathfrak{P}^{\text {int }}+\mathfrak{P}^{\text {ext }}+\mathfrak{P}^{\text {ine }}=0 .
$$

Which is the standard form of principle of virtual powers.

Is the principle of virtual power more general than principle of least Action? First answer: the principle of virtual powers involves differentials which are not exact, in general. Therefore, once fixed the kinematics, the principle of virtual power is actually more general. In both $\mathfrak{P}^{\text {int }}$ and $\mathfrak{P}^{\text {ext }}$ one can include dissipative terms, which cannot, in general, be derived from an Action functional. However, it is not clear if, suitably extending the space of configurations and the set of admissible motions, one can introduce an Action functional also for systems which, in a restricted kinematics, appear as dissipative. Controversies in the literature about this subject are not yet solved.

\section{Hamilton-Rayleigh Approach}

We propose to use the Hamilton-Rayleigh compromise. We introduce an Action functional and a Dissipation Rayleigh functional and, by means of them, we formulate the Principle of Virtual Work. Rayleigh dissipation functional $\mathfrak{R}$ is defined as a linear functional on the set of velocities, not on the set of motions as $\mathfrak{A}$. Therefore, $\delta \mathfrak{R}$ is defined as a linear functional of the variation $\delta \dot{m}$. The principle of virtual works formulated following Hamilton-Rayleigh takes the form: (the lack of the upper dot on RHS is not a mistake!)

$$
\delta \mathfrak{A}(\delta m)=\mathfrak{R}(\delta m) .
$$

\section{Conclusions}

We recall an ancient and useful recipe for building theories for describing effectively physical phenomena:

\section{"In Nomina est Natura Rerum". Anonymous}

This statement (passed to us by the middle age tradition) is formulated for defending mathematical formalism. This sentence claims that it is impossible to talk about any mathematical model without using the appropriate language. So, for instance, it is impossible to say clearly what is the first variation of Action using simply "words" from natural language, 
i.e. without writing integrals on $T$ and, to proceed, we need to give "precisely" names to things. Therefore, to specify precisely how our models are constructed we need to introduce symbols and formulas.

However, we can also say that

\section{"Nomina sunt Consequentia Rerum." Iustinianus, Institutiones Liber II, 7,3}

This because we are not blindly building our mathematical model. We get informations about physics and from these informations we actually formulate our models.

We can finally state that the "old" method of basing the formulation of mathematical models on the variational approach works: indeed it works very well.

\section{Bibliography}

[1] Arnold, V.I., Mathematical Methods of Classical Mechanics, Springer, 2nd edition, May 16, 1989.

[2] Benvenuto, E., La scienza delle costruzioni e il suo sviluppo storico, Sansoni, Firenze, 1981.

[3] Berdichevsky V., Variational Principles of Continuum Mechanics, Springer, 2009.

[4] Bourdin, B., Francfort, G.A., Marigo, J.-J., The variational approach to fracture, J. Elasticity, 91, 1-3, 1-148, 2008. (The paper also appeared as a Springer book: ISBN: 978-1-4020-6394-7).

[5] Colonnetti, G., Scienza delle costruzioni, Torino, Edizioni scientifiche Einaudi, $3^{\text {rd }}$ ed., 1953-57.

[6] Cosserat, E., Cosserat, F., Sur la Théorie des Corps Déformables, Herman, Paris, 1909.

[7] dell'Isola, F., Seppecher, P., The relationship between edge contact forces, double force and interstitial working allowed by the principle of virtual power, Comptes Rendus de l'Academie de Sciences - Serie IIb: Mecanique, Physique, Chimie, Astronomie, vol. 321, pp. 303-308, 1995.

[8] dell'Isola, F., Seppecher, P., Edge Contact Forces and Quasi-Balanced Power, Meccanica, vol. 32, pp. 33-52, 1997.

[9] dell'Isola, F., Madeo, A., Seppecher, P., Boundary Conditions in Porous Media: A Variational Approach, Int. Journal of Solids and Structures, Vol. 46, 3150-3164, 2009.

[10] dell'Isola, F., Madeo, A., Seppecher, P., "Beyond Euler-Cauchy continua". In this book. 
[11] Feynman, R.P., Leighton, R., Sands, M., The Feynman Lectures on Physics: The Definitive and Extended Edition, (Feynman, Leighton, Sands), 3 vv., Addison Wesley, Reading (MA) $2^{\text {nd }}$ ed., 2005.

[12] Francfort, G.A., The chapter in this book by Prof. Gilles Frankfort.

[13] Germain, P., La méthode des puissances virtuelles en mécanique des milieux continus. Premi'ere partie. Théorie du second gradient, J. Mécanique, 12, 235-274, 1973.

[14] Homer. The Iliad. Translated by Murray, A. T., Loeb Classical Library Volumes1. Cambridge, MA, Harvard University Press; London,William Heinemann Ltd., 1924.

[15] Lagrange, J.L., Mécanique Analytique, Editions Jaques Gabay, Sceaux, 1788.

[16] Lanczos, C., The Variational Principles of Mechanics. Toronto: University of Toronto, 1970.

[17] Landau, L.D., Lifshitz, E.M., Quantum Mechanics: Non-Relativistic Theory. Vol. 3 ( $3^{\text {rd }}$ ed.), Pergamon Press, 1977.

[18] Moiseiwitsch, B.L., Variational Principles, Dover, 1966.

[19] Netz, R., Noel., W., The Archimedes Codex: How a Medieval Prayer Book Is Revealing the True Genius of Antiquity's Greatest Scientist, Da Capo press, 2009. See also the website http://www.archimedespalimpsest.org/

[20] Rorres, C., Completing Book II of Archimedes's On Floating Bodies, The mathematical intelligencer, 26,3. Pages 32-42, 2004.

[21] Russo, L., The Forgotten Revolution, Springer Verlag, 2003.

[22] Vailati, G., Il principio dei lavori virtuali da Aristotele a Erone d'Alessandria, Scritti (Bologna, Forni, 1987), vol. II, pp. 113-128. Atti della R. Accademia delle Scienze di Torino, vol. XXXII, adunanza del 13 giugno 1897, quaderno IG (091) 75 I - III. 1987. 